\title{
La gestión del turismo comunitario para el desarrollo local de la parroquia Salasaca, Ecuador
}

Community tourism management for local development of Salasaca parish, Ecuador

Esther del Carmen Mullo Romero. ${ }^{1}$, Yasser Vázquez Alfonso. ${ }^{2}$

\begin{abstract}
.
DOI: https://doi.org/10.33262/cienciadigital.v3i4.902

Thinking about community tourism based on the essential needs of the local population is the fundamental basis for its proper planning and development. The research was developed in the parish of Salasaca belonging to the Pelileo canton, in the province of Tungurahua, aimed to enhance the management of Community Tourism for local development. A diagnosis was made on the current situation of the locality through the use of the SWOT matrix and the SWOT 1.0 software. The information was collected through the application of a survey to 340 community members and 22 managers directly linked to the tourism activity. The information was processed and analyzed using the Multivariate Multiple Correspondence Analysis (ACM) technique. implemented, in the SSPS software version 23.0. The main limitations of the community are identified and analyzed, which indicate that internal weaknesses must be improved, taking advantage of external opportunities. Validation of the strategy was carried out using the Delphi, Anochi and Iadov methods, to experts and users, who showed high satisfaction results. Among the conclusions it can be seen that an adequate management for community tourism allows the takeoff of the communities and a local development, producing a revival of the almost disappeared cultures.
\end{abstract}

${ }^{1}$ Universidad de la Habana, Cuba, emulloromero@gmail.com

${ }^{2}$ Universidad de la Habana, Facultad de Turismo, Cuba, yalfos1 @ gmail.com 
Keywords: Local community development, Community Tourism, Tourism Management, Culture.

\section{Resumen.}

Pensar en el turismo comunitario a partir de las necesidades esenciales de la población local, es la base fundamental para su adecuada planificación y desarrollo. La investigación se desarrolló en la parroquia de Salasaca perteneciente al cantón Pelileo, en la provincia de Tungurahua, tuvo como objetivo potenciar la gestión del Turismo Comunitario para el desarrollo local. Se realizó un diagnóstico sobre la situación actual de la localidad a través del empleo de la matriz DAFO y el software DAFO 1.0. Se recopiló la información a través de la aplicación de una encuesta a 340 miembros de la comunidad y 22 directivos vinculados directamente a la actividad turística, siendo procesada y analizada la información mediante la técnica de la Estadística Multivariada de Análisis de Correspondencia Múltiple (ACM), implementada, en el software SSPS versión 23.0. Se identifican y analizaron las principales limitantes de la comunidad, las cuales señalan que se debe mejorar las debilidades internas, aprovechando las oportunidades externas. La validación de la estrategia se realizó mediante los métodos Delphi, Anochi y Iadov, a expertos y usuarios, que mostraron resultados de alta satisfacción. Entre las conclusiones se puede apreciar que una adecuada gestión para el turismo comunitario permite el despegue de las comunidades y un desarrollo local, produciendo un reavivamiento de las culturas casi desaparecidas.

Palabras claves: Desarrollo local comunitario, Turismo Comunitario, Gestión Turística, Cultura.

\section{Introducción.}

El turismo comunitario está apareciendo con fuerza en la región andina y esencialmente está basado en la comunidad local y en la gestión del territorio, es una modalidad turística donde la comunidad local participa de forma activa en este tipo de actividades, la gestión de los recurso permite generar riqueza en las áreas rurales de los países de Latinoamérica, a través de la participación de la comunidad local en la gestión turística, de forma tal que los beneficios obtenidos repercutan en la propia comunidad. Además, es capaz de promover el desarrollo integral de las comunidades tratando de reducir la pobreza a través de la generación de empleo y la obtención de ingresos complementarios, evitando los movimientos migratorios (Inostroza V, 2010).

Las investigaciones desarrolladas en la temática del turismo comunitario se evidencian en varios países latinoamericanos, tal es el caso de la República del Perú y Bolivia, desarrollado por (Olivera 
, Negrón, Muñoz , \& Paucar, 2012); (Sepúlveda, Basurto, \& Vizcarra, 2010) y (Pacheco, Carrera , \& Almeida, 2011), en donde se evidencia que en la actualidad existen algunas organizaciones y agrupaciones que reflejan que la participación de las comunidades han tomado diversas manifestaciones, por ejemplo en el lago Titicaca y en el Valle Sagrado de los Incas, estas actividades se desarrollan a nivel comunal, donde trabajan grupos privados en conjunto con los municipios, sin la participación de representantes del ministerio a cargo del turismo, siendo uno de los problemas que enfrentan los diferentes actores relacionados con el turismo, la falta de mecanismos de comunicación y de intercambio son la razón por la cual han propuesto la creación de una Red de Turismo Sostenible (RedTurs) que agrupa a los distintos sectores interesados en el desarrollo. Costa Rica es uno de los destinos de Latinoamérica más interesantes, debido a que el turismo comunitario ha sido el resultado de una evolución que ha ido desde un planteamiento de aprovechamiento de la riqueza natural hacia una visión de carácter social, siendo sus inicios la observación y la participación en proyectos agrícolas de cooperativas, a medida que han ido avanzando se combina con otras facetas culturales y sociales, hasta llegar al turismo rural comunitario que se conoce hoy en día (Trejos, 2009).

Por otro lado, en Brasil el turismo comunitario comenzó a desarrollarse en el Estado de Santa Catarina, pero en la actualidad se desarrollan actividades en todo el país, aunque con mayor medida en los estados del sur (Bauman, 2003), es a partir de la coordinación del desarrollo alcanzado por estas comunidades que a través de la sociedad entre comunidades, el Instituto Laboratorio de Gestión de Organizaciones que promueven el Eco-desarrollo y la Universidad Regional de Blumenau que se viene desarrollando, desde el año 2006, una metodología educativo participativa, basada en la investigación - acción, la que se conoce actualmente como turismo de base comunitaria y solidaria (Instituto Latinoamericano y del Caribe de Planificación Económica y Social- ILPES, 2003).

En el territorio ecuatoriano, el turismo comunitario aparece a finales de los años 70’s, en respuesta a la fuerte sensación de exclusión de las comunidades, que se manifestaron como un objeto de apreciación, más que un sujeto en el desarrollo del turismo en sus territorios (Cabanilla, 2012).

El turismo comunitario se articula a nivel nacional alrededor de dos redes: la Red Ecoturística Nacional y la Asociación Comunitaria de Turismo Rural, quienes desarrollan programas de apoyo, promoción, formación y de calidad dirigidos a las asociaciones y cooperativas socias (Nel-Lo, 2008).

Para Cañada (2018), las actividades de turismo comunitario en Latinoamérica se han desarrollado por múltiples vías, he aquí algunas de ellas:

- Cooperativas y familias campesinas que buscan ampliar, diversificar y complementar ingresos.

- Comunidades indígenas que realizan actividades complementarias para mejorar su economía y bienestar. 
- Organizaciones ambientalistas de carácter comunitario que tratan de generar ingresos para dar sostenibilidad a sus labores de conservación de los recursos naturales.

- Comunidades posconflicto que intentan su reinserción a la vida civil y mantener la memoria.

- Grupos de mujeres que incrementan los recursos con los cuales atienden las necesidades familiares y que, en algunos casos, favorecen su autonomía.

- Pequeños negocios privados articulados asociativamente, que tratan de potenciar y complementar su oferta.

Estas actividades son definidos como iniciativas empresariales que "en una lógica de mutuo beneficio incorporan en sus cadenas de valor a comunidades de bajos ingresos"; de este modo contribuyen a que las empresas "amplíen sus segmentos de mercado hacia sectores de la población de bajos ingresos, a la vez que promueven que las familias en situación de pobreza aprovechen las oportunidades que ofrece el mercado y la dinámica del sector empresarial" (SNV y WBCSD, 2010).

El Turismo Comunitario tiene sus inicios en la provincia Tungurahua a partir del 2006 según Aristegui (2016) y las comunidades pioneras fueron Salasaca, Quillán, Cunukyaku e Illawa Chico, quienes propusieron actividades turísticas comunitarias, en aquellos tiempos la comunidad no tenía claro el concepto de organización comunitaria, el involucramiento y la responsabilidad que repercute dicha actividad. Actualmente el desarrollo del Turismo Comunitario en la provincia se ha visto precario por el desinterés de la población misma y las autoridades locales, y un manejo empírico de los sitios turísticos.

Las investigaciones realizadas en el contexto de la Parroquia Salasaca por: (Pérez, 2012; Solís 2016 y Masaquiza, 2017), resaltan la necesidad de gestionar y potenciar el desarrollo del Turismo Comunitario y plantean las siguientes problemáticas:

- Falta de políticas que incentiven los comercios culturales.

- Desinterés de las autoridades en potenciar la actividad turística.

- $\quad$ Escaso presupuesto económico gubernamental.

- $\quad$ Mínimo aprovechamiento de los recursos ambientales (naturales y culturales).

- Baja promoción turística de la comunidad a través de la diversidad cultural.

- Desvaloramiento de las culturas indígenas.

- $\quad$ Poca difusión de las manifestaciones culturales.

- $\quad$ Poca práctica del idioma kichwa.

- Debilidad de la identidad en la parroquia Salasaca.

- Los jóvenes adaptan culturas diferentes.

- Inexistencia de educación cultural.

- Malas estrategias de educación cultural.

- $\quad$ Escaso turismo en las fiestas. 
- Desconocimiento de las fiestas populares.

- Los habitantes poseen escasa cultura turística.

- Información empírica del turismo cultural.

- Inexistencia de capacitaciones sobre el turismo cultural.

- $\quad$ Poco ingreso económico en la comunidad por concepto de turismo.

Para darle cumplimiento a las anteriores problemáticas se enuncia el objetivo de esta investigación que es potenciar la gestión del turismo comunitario para el desarrollo local de la parroquia Salasaca, Ecuador.

\section{Materiales-métodos.}

La investigación se realiza en el centro de los Andes del Ecuador, en la provincia de Tungurahua, a $13 \mathrm{Km}$ del cantón Ambato, al oeste del cantón Pelileo, cerca al cerro Teligote, se encuentra la comunidad quichua Salasaca. Sobre su origen consideran que son descendientes de antiguos mitimaes procedentes de Bolivia. Su extensión aproximada es de 14,3 Km2, Tiene como límites: Al Norte la parroquia el Rosario y Picaihua, Al Sur la parroquia Benítez y Pelileo, Al Este García Moreno y Al Oeste la parroquia Totoras. Su ubicación astronómica corresponde a: 7845 68” longitud occidental; y $1^{\circ} 10^{\prime} 58^{\prime \prime}$ latitud Sur, se encuentra ubicada en:

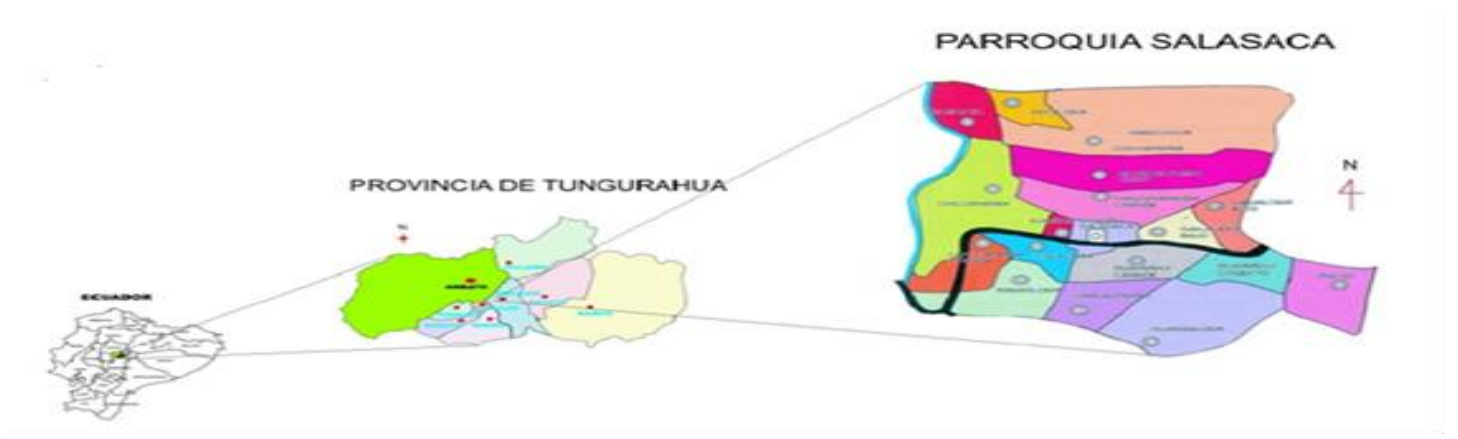

Figura 1: Ubicación Geográfica de la parroquia Salasaca.

Fuente: Elaboración propia.

\section{Metodología.}

El procedimiento investigativo de la investigación se fundamenta en la investigación cualitativa y hace uso de a los métodos y técnicas de investigación-acción participativa, así como de técnicas analíticas apropiadas para estudios de planificación del turismo comunitario. Con el cual se alcanza explorar las relaciones sociales y describir la realidad del turismo comunitario. Tomando como punto central el estudio de caso realizado en la parroquia Salasaca.

Durante el proceso de investigación se requirió la aplicación de los siguientes métodos teóricos: 
Histórico-Lógico: Para conocer el comportamiento y evolución de las diferentes posiciones respecto a la gestión del turismo comunitario basado en la diversidad cultural que resulta de interés para la investigación.

Análisis-Síntesis: Permitieron llegar a conclusiones sobre la base de las diferentes fuentes, a partir del estudio realizado del tema objeto de investigación y apreciar su valoración desde diferentes posiciones teóricas.

Inductivo-deductivo: Para transitar de lo general a lo particular y viceversa, al establecer los nexos entre los diferentes elementos relacionados con la gestión del turismo comunitario basado en la diversidad cultural, a partir de los indicadores y dimensiones obtenidas del objeto de investigación.

Modelación: Para representar las características y relaciones fundamentales del objeto y elaborar la estrategia como resultado.

Enfoque de sistema: Con el objetivo de lograr la interacción entre los diferentes elementos que conforman la estrategia elaborada.

Además, se aplicaron los siguientes métodos empíricos:

Consulta de documentos: Fue empleado en la revisión de la literatura especializada y los documentos rectores de la política ecuatoriana sobre la gestión del turismo comunitario y para extraer la información necesaria relacionada con el objeto de investigación.

Observación (directa, abierta, sistemática y participante): Para obtener información de los niveles de desarrollo durante la gestión del turismo comunitario basado en la diversidad cultural.

Encuestas: Para extraer información sobre el conocimiento de los miembros de la comunidad, especialistas y dirigentes acerca del problema de investigación.

Entrevistas: Empleada para obtener información a partir de la experiencia de dirigentes del turismo comunitario, acerca del problema de investigación.

Como métodos estadísticos-matemáticos se aplicaron:

Estadística Descriptiva: Se utiliza para el procesamiento de los resultados obtenidos del diagnóstico y la evaluación práctica de la estrategia mediante tablas, gráficos y estadígrafos.

Estadística multivariada: se utilizó para el estudio multivariado del objeto de investigación a través del Análisis de los Correspondencia Múltiple (ACM).

Concepción de la Investigación 
En el trabajo de investigación se planea cinco fases que aparecen en la figura 1 las cuales son:

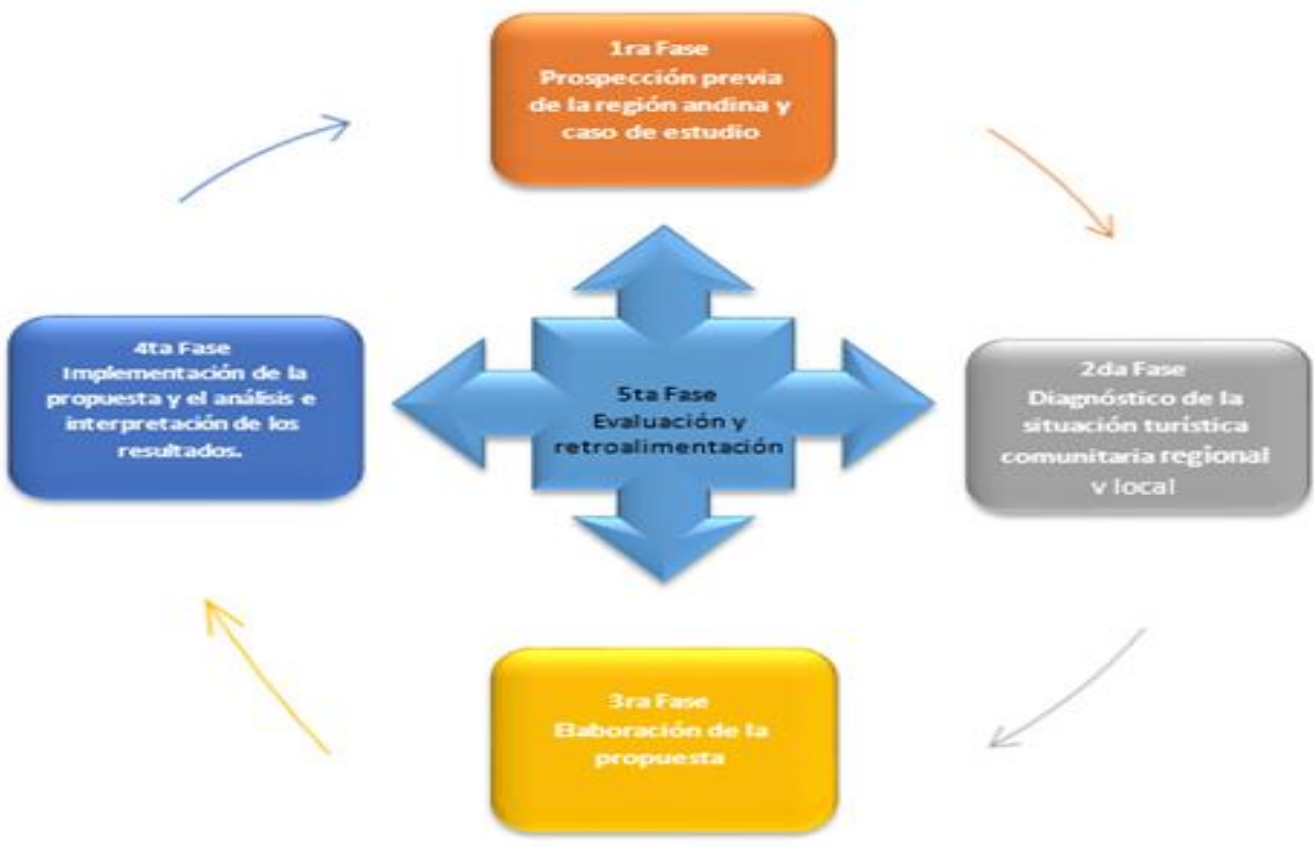

Figura 2. Fases de la investigación.

Fuente: Elaboración propia.

\section{Resultados.}

Para alcanzar un diagnóstico objetivo del estado actual de las estrategias que permitan una gestión adecuada de turismo comunitario, tomando en cuenta el desarrollo local, se seleccionó varios instrumentos para comprobar los niveles de desarrollo de cada indicador correspondientes a las dimensiones constituidas en el gráfico anterior. Se procede, a continuación, a la presentación de los resultados obtenidos a partir de los métodos empíricos aplicados, sobre la base de los instrumentos utilizados.

Como resultado de la técnica del diagnóstico participativo a miembros representativos de la comunidad se pudo determinar los factores externos e internos del caso de estudio, los que se muestran a continuación:

Ambiente interno

\section{Fortalezas}

1. Salasaca posee su propia cultura, saberes y prácticas productivas, que se transmiten de generación en generación; por lo que se ha mantenido a través de los tiempos

2. Zona con potencial en turismo comunitario por su riqueza cultural y paisajista (Teligote, Kinlliurko, Nitón Cruz, Cruz Pamba) 
3. Presenta una ubicación estratégica junto a la vía principal que comunica con la región oriental

4. Zona de hábiles e ingeniosos artesanos que confeccionan tapices para el mercado local, nacional e internacional.

5. Líderes profesionales y capacitados con diversas especializaciones, desempeñan funciones públicas, directivos en organizaciones sociales en el ámbito local y nacional.

6. Existe la dotación de infraestructura y servicios básicos en la mayoría de las comunidades.

7. Participación activa de los miembros de la comunidad en las asambleas y mingas.

8. Debilidades

9. Limitada infraestructura comunitaria para desarrollar el turismo.

10. Poca planificación del turismo comunitario en las comunidades de Salasaca.

11. Inexistencia de esquemas turísticos para el desarrollo y comercialización de los productos turísticos

12. Limitado desarrollo del servicio hotelero y gastronómico en sectores turísticos.

13. Desconocimiento de la demanda real y potencial, de los agentes involucrados en la oferta turística, y de las relaciones económicas entre los agentes.

14. Poca gestión, en el desarrollo de productos, servicios, promoción y Comunicación del Destino Turístico

15. Presencia de un gran movimiento migratorio interregional e internacional, fenómeno que se refleja en la población joven masculina por falta de fuentes de trabajo y desorganización familiar.

16. Baja capacidad productiva de los suelos por erosión e inadecuadas prácticas agrícolas occidentales.

17. Presencia de una larga cadena de intermediarios, en el proceso de comercialización que reduce significativamente el valor agregado de los productos artesanales.

18. Baja capacidad de inversión de las familias para mejorar la producción agropecuaria y artesanal.

Ambiente externo

\section{Oportunidades}

1. Reconocimiento a las nacionalidades y pueblos como parte del Estado Ecuatoriano, en su constitución política.

2. Apoyo de instituciones de cooperación a comunidades y organizaciones indígenas.

3. Presencia de turistas extranjeros y nacionales que dinamizan el turismo y la feria artesanal de la localidad.

4. Gran interés por parte de investigadores para realizar proyectos, gracias a que Salasaca es un pueblo ancestral por su riqueza cultural

5. Presencia de miembros del pueblo Salasaca en cargos directivos de organizaciones indígenas nacionales y en diversas instituciones gubernamentales, que favorece la gestión de proyectos para el sector. 


\section{Amenazas}

1. El proceso de globalización tendiente a una homogenización, sin respetar las particularidades culturales.

2. Las pugnas y la falta de un auténtico liderazgo en organizaciones nacionales influyen en el fraccionamiento de las estructuras organizativas populares.

3. Las ofertas clientelares de los políticos crean falsas expectativas en la población.

4. La presencia de varias organizaciones cooperantes realizando un trabajo aislado en la misma zona de influencia y con un carácter asistencialista.

Dentro del diagnóstico turístico la principal limitante para que el turismo comunitario no haya alcanzado su pleno desarrollo en la comunidad, es que ha carecido de un proceso de planeación previo, en la que se apliquen estrategias adecuadas de promoción, marketing, desarrollo del destino que den a conocer todos los atractivos turísticos que poseen, Schaller (2010), considera que es un "Instrumento que permite determinar en conjunto con la comunidad el tipo de proyectos que se busca atraer y cuáles serían los emplazamientos territoriales y las condiciones de operatividad, velando además por la sostenibilidad de los mismos".

Para la gestión turística se identificó que no existe registro documentado del plan o proyecto de planificación estratégica específica para el turismo, existe solamente de manera general para todos los aspectos de la comunidad.

En la gestión del marketing y proyección, el marketing en la parroquia Salasaca para el turismo comunitario es muy débil y casi nulo, no existe canales definidos de comercialización, los medios de promoción y tampoco los costos y beneficios de los servicios, además se aprecia una carencia fuerte en el análisis de los emprendedores de la comunidad, lo que ha impedido que se realicen capacitaciones en el área turística, así como en la educación y sensibilización limitando así la calidad de vida de la población y de aquellos que trabajan en el turismo comunitario causando de a poco una pérdida de la cultura.

Para el diagnostico se analizó la dimensión del Plan Estratégico en el cual se determinó la inexistencia de acciones que indiquen claramente cuál es la misión o función del turismo comunitario en Salasaca y no se han realizado investigaciones del desarrollo de productos turísticos comunitarios en ese lugar tomando en cuenta la opinión de las autoridades de la comunidad.

Una vez aplicada la encuesta a los miembros de la comunidad se llega a determinar que, en mayor proporción las acciones turísticas en Salasaca se realizan rara vez, como son:

Dimensión Capacidad de carga para los atractivos 
Del total de la población encuestada se mencionó que rara vez hay cuidado a los recursos y que estos con el pasar del tiempo pueden ser potencialmente dañados por el turismo (instauración de límites de cantidad de personas en un momento determinado, etc.)

En su mayoría comentan que rara vez existe una buena estimación de la oferta y demanda turística y una gestión acorde a los requerimientos que presentan

\section{Dimensión Planificación turística}

- Se menciona que rara vez existe la posibilidad de optar a programas de financiamiento para mejorar la calidad de productos y/o servicios.

- Hay iniciativas para el fomento de las visitas hacia los diversos atractivos turísticos (City Pass, descuentos, etc.) que rara vez se dan.

- La mayoría señala que rara vez que existe la participación local para desarrollar la oferta turística alojamiento, alimentación y recreación.

- Del total encuetados señalan que rara vez se realizan alianzas con centros de formación especializados en turismo para mejorar la calidad turística.

- Rara vez existe una oferta suficiente de alojamiento, gastronomía y recreación en la comunidad.

- $\quad$ Dimensión manifestaciones culturales y calidad de servicios

- La comunidad quiere y está dispuesta a dar a conocer su cultura, siempre en su gran mayoría.

- Para la mayoría de involucrados los productos y servicios siempre cuentan con una exitosa gestión turística los mismos que son reconocidos públicamente.

De lo anterior se puede definir las variables que intervienen en el turismo comunitario, tales como: calidad de los productos y servicios, mantenimiento de recursos turísticos, fomentos de atractivos, alojamiento, alimentación y recreación; demanda y oferta de los servicios para mejorar la calidad, tienen una aplicación limitada en el turismo comunitario de Salasaca, por tal razón los planificadores lo han considerado como debilidades del sector. Existe una escasa participación de la comunidad para compartir sus conocimientos relacionados con su cultura y las tradiciones del pueblo de Salasaca para la aceptación de ésta a nivel nacional e internacional.

Como resultado de la sumatoria de cuadrantes se obtuvo que el cuadrante de mayor valor es el de debilidad - oportunidad, de esta manera la parroquia de Salasaca requiere de estrategias adaptativas (min - maxi), ver figura 2.2:

\section{Estrategia Adaptativa}

Las estrategias de adaptación, tienen la finalidad de mejorar las debilidades internas, aprovechando las oportunidades externas. La organización a la cual el entorno le brinda ciertas oportunidades, pero no las puede aprovechar por sus debilidades, podría decidirse a invertir recursos para 
desarrollar el área deficiente y así poder aprovechar la oportunidad. Entre las estrategias adaptativas, que se utilizan con mayor frecuencia se encuentran las siguientes:

a) Las estrategias de adaptación que se conoce como "Penetración en el Mercado", está dirigida a aumentar la participación en el mercado de los productos o servicios actuales, por medio de un esfuerzo mayor para la comercialización. Esta estrategia muchas veces se usa sola o también en combinación con otras. La penetración en el mercado incluye aumentar la cantidad de vendedores, elevar el gasto publicitario, ofrecer muchas promociones de ventas con artículos en función de la necesidad de los clientes o usuarios externos (González; 2016).

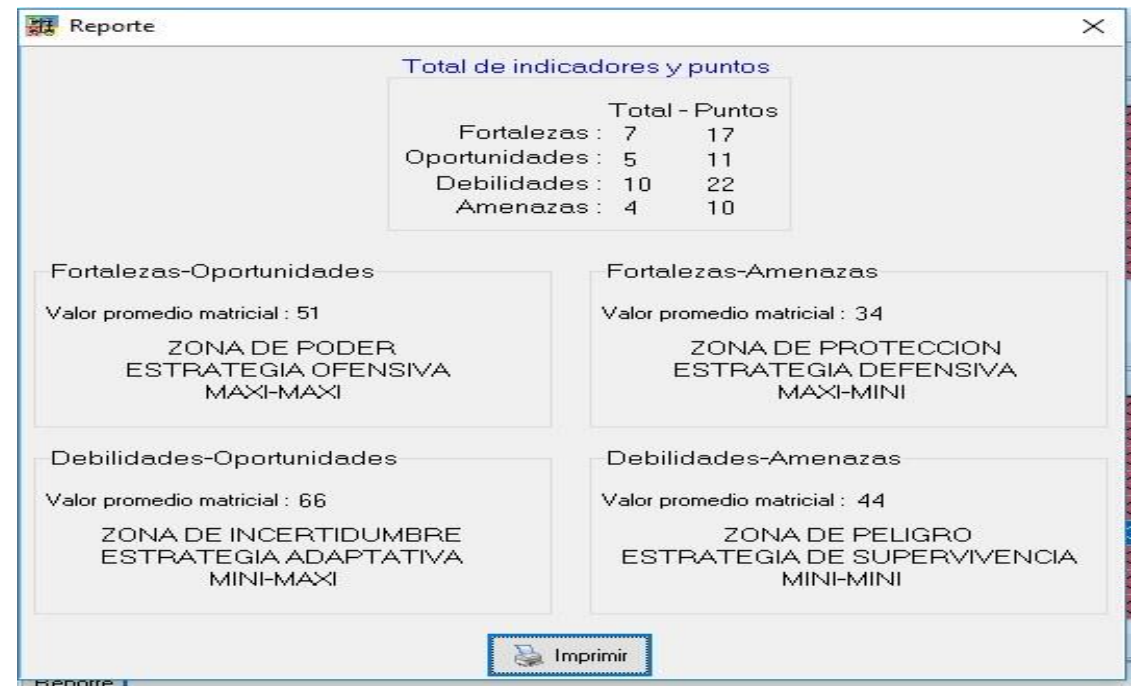

Figura 3. Resultado del procesamiento de la matriz DAFO a través del software DAFO 1.0. Fuente: Elaboración propia.

Con este resultado se puede demostrar mediante el método de impactos cruzados que analiza las interrelaciones mutuas existentes entre las variables claves identificadas, se define el problema estratégico, los objetivos estratégicos y las acciones a seguir, las cuales deben ser factibles de materializar en un período de corto plazo. Es importante que en Salasaca se minimicen las debilidades tales como: 1) Inexistencia de esquemas turísticos para el desarrollo y comercialización de los productos turísticos; 2) Poca planificación del turismo comunitario en las comunidades de Salasaca; 3) Limitada infraestructura comunitaria para desarrollar el turismo; 4) Limitado desarrollo hotelero y gastronómico en sectores turísticos, y; 5) Desconocimiento de la demanda real y potencial, de los agentes involucrados en la oferta turística, y de las relaciones económicas entre los agentes; mediante el aprovechamiento de las oportunidades tales como: 1) Presencia de turistas extranjeros y nacionales que dinamizan el turismo y la feria artesanal de la localidad; 2) Gran interés por parte de investigadores sociales por realizar investigaciones gracias a que Salasaca es un pueblo ancestral y por su riqueza cultural, y; 3) Apoyo de instituciones de cooperación a comunidades y organizaciones indígenas. 
Para realizar un análisis más integral de las variables que componen la encuesta se empleó el método de Análisis de Correspondencia Múltiple (ACM). Los resultados de la aplicación de este método aparecen en la figura 2.3 y 2.4, refleja los valores del Alfa de Cronbach en la dimensión 1 y 2, los cuales están dentro del rango de fiabilidad de la aplicación adecuada del método según (Hair, Anderson, \& Tatham, 1999). La dimensión 1 explica aproximadamente un 33\% de la variabilidad y las variables que presentan más pesos son:

Desarrollo de productos y servicios turísticos (DPST), Identificación de oportunidades para el desarrollo turístico(IOPDT), Identificación de los procesos planificación y ordenamiento territorial (IPPOT), Presencia mejorada en la calidad de vida de la población (PMCVP), Fomento de la cultura turística (FCT). La dimensión 2 explica un $29 \%$ de la variabilidad y las variables que más pesos tienen son: Considera usted que la cultura, tradiciones creencias, formas de convivencia, y costumbres, en las relaciones sociales de la comunidad se han modificado con el desarrollo del turismo comunitario (CCTSCDTC), Identificación de los problemas y causas de la actividad turística (IPCAT) y Frecuentemente se ejecutan programas de monitoreo y evaluación para el turismo comunitario (FEPMTC). Con respecto al comportamiento de los criterios que dan los encuestados se muestra en la gráfica del anexo, que la gran mayoría de los especialistas poseen criterios diferentes sobre la percepción que tienen sobre la gestión integral participativa para el turismo comunitario basado en la diversidad cultural.

Los resultados obtenidos con la aplicación de las encuestas, evidencian la necesidad de gestionar el desarrollo del turismo comunitario de la parroquia Salasaca en el contexto ecuatoriano.

\begin{tabular}{|c|c|c|c|c|}
\hline \multicolumn{5}{|c|}{ Resumen del modelo } \\
\hline \multirow[b]{2}{*}{ Dimensión } & \multirow[b]{2}{*}{$\begin{array}{l}\text { Alfa de } \\
\text { Cronbach }\end{array}$} & \multicolumn{3}{|c|}{ Varianza explicada } \\
\hline & & $\begin{array}{c}\text { Total } \\
\text { (Autovalores) }\end{array}$ & Inercia & $\begin{array}{l}\% \text { de la } \\
\text { varianza }\end{array}$ \\
\hline 1 & 978 & 30,450 & ,331 & 33,098 \\
\hline 2 & ,973 & 26,820 & ,292 & 29,152 \\
\hline Total & & 57,270 & ,622 & \\
\hline Media & ,976 & 28,635 & ,311 & 31,125 \\
\hline
\end{tabular}

Figura 4. Resultado de la aplicación ACM.

Fuente: Elaboración propia. 


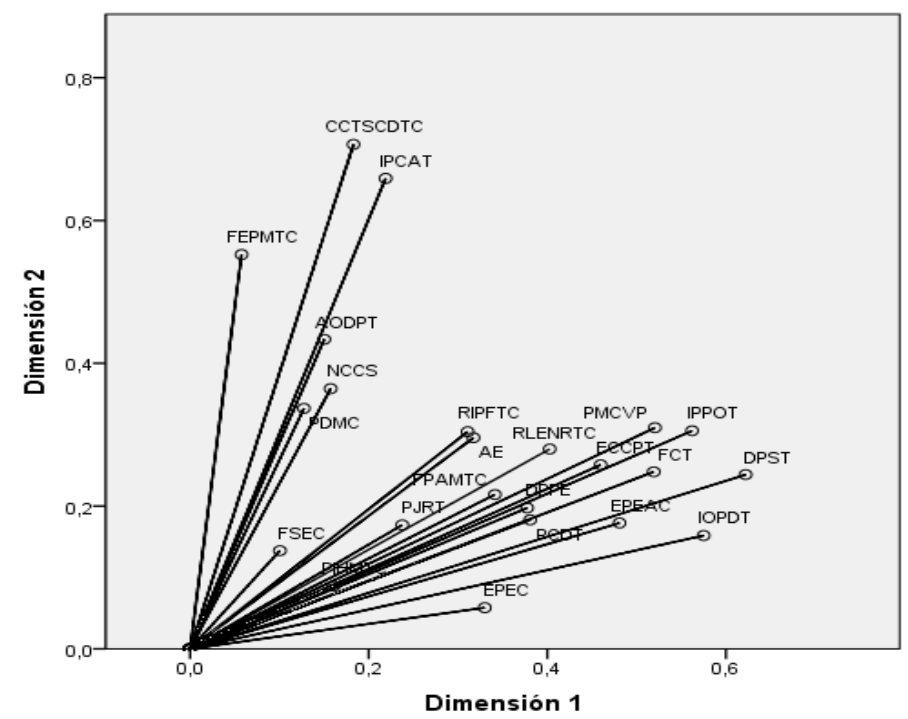

Figura 5. Gráfico de Biplot de las variables.

Fuente: Elaboración propia.

\section{Conclusiones.}

- La culminación de esta investigación ha permitido arribar a las conclusiones siguientes:

- Los referentes teóricos asumidos sobre el turismo comunitario, permitieron a la autora de la investigación tomar posiciones con respecto al estudio de la gestión del turismo comunitario en la comunidad Salasaca.

- De la aplicación de los diferentes métodos, técnicas y herramientas en el diagnóstico de la gestión del turismo comunitario se evidenció que hay inadecuados niveles de planificación para el turismo comunitario, inexistencia de esquemas turísticos para el desarrollo y comercialización de los productos turísticos; poca planificación del turismo comunitario en las comunidades de Salasaca; limitada infraestructura comunitaria para desarrollar el turismo; limitado desarrollo hotelero y gastronómico en sectores turísticos, y desconocimiento de la demanda real y potencial, de los agentes involucrados en la oferta turística, y de las relaciones económicas entre los agentes.

\section{Referencias bibliográficas.}

Bauman, Z. (2003). Community. Seeking safety in an insecure world. London: Polity Press.

Cabanilla, E. (2012). Turismo Comunitario en Ecuador: contenidos y relaciones que pensar en relación al acontecimineto turístico. Pasos, 109 - 149.. 
Cañada, E. (28 de Mayo de 2018). La comercialización del turismo comunitario en América Latina. Obtenido de https://www.researchgate.net/publication/284284507.

González Alva, E. D. (06 de Mayo de 2016). Blogger. Obtenido de http://imi92dgonzalez.blogspot.com/2016/06/tarea-5-estrategias-ofensiva-defensiva.html

Inostroza V , G. (2010). Aportes para un modelo de gestión sostenible del turismo comunitario en la región andina. Gest. tur. (Valdivia).10, 77-90

Masaquiza, J.B (2013). Las manifestaciones culturales y la identidad del pueblo Salasaka. Tesis presentada para optar por el título de Licenciado en Comunicación Social. Facultad de Jurisprudencia y Ciencias Sociales. Universidad Técnica de Ambato. Ambato. Ecuador.235p.

Masaquiza, D.E (2017). El Turismo Comunitario y su aporte al desarrollo turístico de la parroquia de Salasaca cantón Pelileo, provincia de Tungurahua. Tesis presentada para optar por el título de Licenciado en Turismo y Hotelería. Facultad de Ciencias Humanas y de la Educación. Universidad Técnica de Ambato. Ambato. Ecuador. 147p.

Nel-Lo, M. (2008). Organización y características del turismo rural comunitario en Costa Rica. Anales de Geografía, 28(2), 167-188.

Olivera , M., Negrón , C., Muñoz , J., \& Paucar, E. (2012). Planteamineto estrategico para el turismo rural comunitario en Cuatro Lagunas. Cusco. Cusco.

Pacheco, V., Carrera , P., \& Almeida, K. (2011). Propuesta metodológica para la evaluación de la factibilidad de proyectos de turismo comunitario. Cusco.

Pérez, E.P (2012). El desconocimiento de la fiesta popular de los Caporales de la comunidad indígena de Salasaka y su incidencia en la comunicación cultural durante el 2010. Tesis presentada para optar por el título de Licenciado en Comunicación Social. Facultad de Jurisprudencia y Ciencias Sociales. Universidad Técnica de Ambato. Ambato. Ecuador.125p.

Schaller, D. (2010). indigenous Ecotourism and Sustainable Development. The Case of Rio Blanco, Ecuador.Estados Unidos: Univesity of Minnesota.

Sepúlveda, H., Basurto, R., \& Vizcarra, Y. (2010). plan estratégico para el desarrollo del turismo rural comunitario en la región Cusco. Cusco.

SNV y WBCSD. (2010). Negocios Inclusivos: creando valor en América Latina. s/c: Netherlands Development Organization (SNV) - Consejo Empresarial Mundial para el Desarrollo Sostenible (WBCSD). 48-68. 
Trejos, B. (2009). Redes de apoyo comunitario al turismo comunitario en Costa Rica, TURyDES. Revista de Investigación en Turismo y Desarrollo Local, 2 (6), 1-15. 
PARA CITAR EL ARTÍCULO INDEXADO.

Mullo Romero, E. del C., \& Vázquez Alfonso, Y. (2019). La gestión del turismo comunitario para el desarrollo local de la parroquia Salasaca, Ecuador. Ciencia Digital, 3(4), 122-137. https://doi.org/10.33262/cienciadigital.v3i4.902

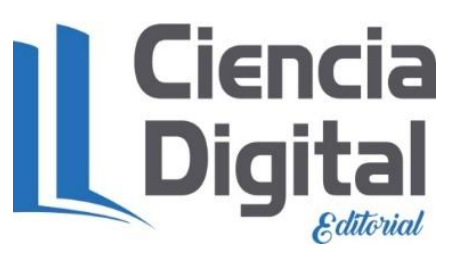

El artículo que se publica es de exclusiva responsabilidad de los autores y no necesariamente reflejan el pensamiento de la Revista Ciencia Digital.

El artículo queda en propiedad de la revista y, por tanto, su publicación parcial y/o total en otro medio tiene que ser autorizado por el director de la Revista Ciencia Digital.
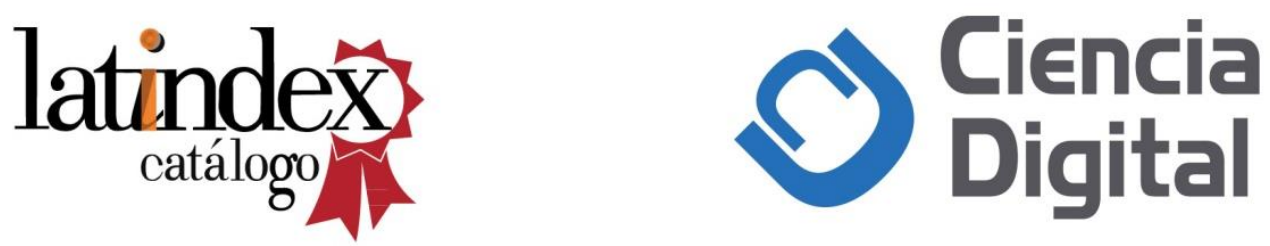